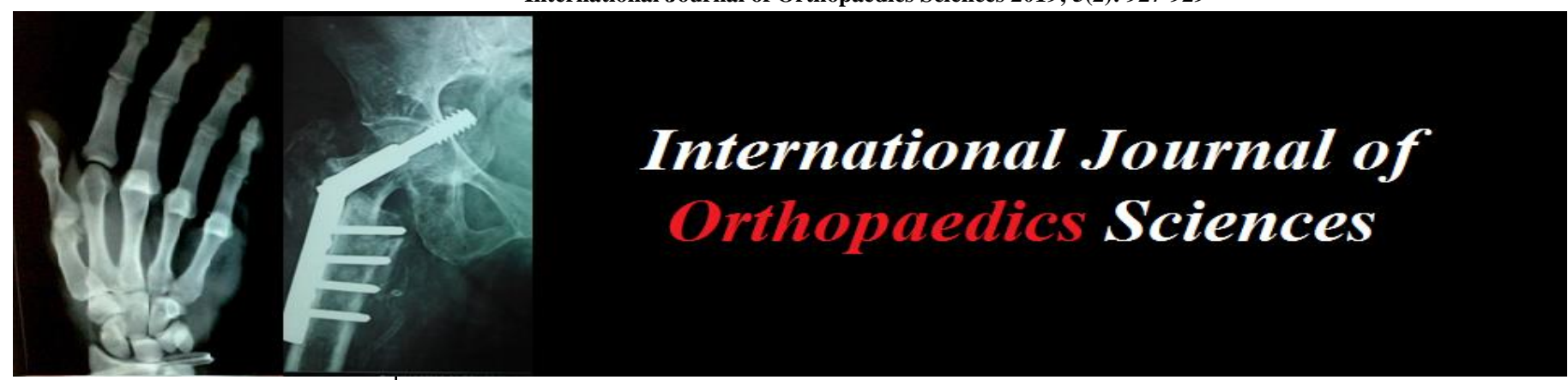

ISSN: $2395-1958$

IJOS 2019; 5(2): 927-929

(C) 2019 IJOS

www.orthopaper.com

Received: 06-02-2019

Accepted: 10-03-2019

Shingati Muhammad Hashim

Associate Professor,

Rajarajeshwari Medical College

Bangalore, Karnataka, India

Dr. Ullas Mahesh

Assistant Professor, Department

of Orthopaedics, Sidhartha

Medical College Begur,

Karnataka, India

Sharath S Jadamali

Post Graduate, Rajarajeshwari

Medical College Bangalore,

Karnataka, India
Correspondence

Dr. Ullas Mahesh

Assistant Professor, Department

of Orthopaedics, Siddhartha

Medical College Begur,

Karnataka, India

\section{Clinical and radiological outcome in cases of posterior lumbar interbody fusion for lumbar spondylolisthesis}

\author{
Shingati Muhammad Hashim, Dr. Ullas Mahesh and Sharath S Jadamali
}

DOI: https://doi.org/10.22271/ortho.2019.v5.i2n.113

\section{Abstract}

Introduction: Spondylolisthesis is the subluxation of a vertebral body over another in the sagittal plane. It represents a particular and relatively frequent mechanism of intervertebral instability. This pathology can be caused by ligamentous laxity, a defect in the pars interarticularis.

Results: There was significant reduction in the postoperative pain and disability at 6 months follow up in back and leg and significant reduction of slippage radiological and significant bony union.

Conclusion: Decompression primarily relieves radicular symptoms and neurogenic claudication whereas fusion primarily relieves back pain by elimination of instability. The addition of posterofusion instrumentation (Pedicle screws and cage) enhances the ability to obtain a solidarthrodesis, Posterior Lumbar interbody fusion is a excellent procedure for lumbar spondylolisthesis achieved a significant reduction in low back pain and claudication pain and significant amount of radiological reduction and union.

Keywords: posterior lumbar interbody fusion, VAS score (Visual analogue score), radiological slippage

\section{Introduction}

Spondylolisthesis is the subluxation of a vertebral body over another in the sagittal plane. It represents a particular and relatively frequent mechanism of intervertebral instability. This pathology can be caused by ligamentous laxity, a defect in the pars interarticularis, previous surgery, or may be traumatic and occurs in up to $5 \%$ of the general population and affects all ages. Lumbar Spondylolisthesis is a common cause for lower-back pain, radiculopathy, and neurogenic claudication among the adult population, The nonoperative treatment modalities of degenerative spondylolisthesis have remained relatively unchanged during the past two decades, The surgical treatment of spondylolisthesis is indicated for cases of neurogenic claudication, intractable radicular pain, severe low back pain, presence of neurological symptoms, failure of conservative management, radiological instability, progressive worsening of the listhesis, Meyerding grade III and IV listhesis, and spondyloptosis. The ideal surgical treatment remains controversial. Lumbar interbody fusion is the most reliable fusion technique currently available for the lumbar spine as these constructs are biomechanically stronger, provide axial support with less graft subsidence or collapse comparing to those with posterolateral arthrodesis, and produce a better biologic fusion in lordotic alignment ${ }^{[1-5]}$. In theory interbody fusion provides several advantages when compared with other fusion techniques as it immobilizes the painful degenerated spinal segments, decompress the nerve roots, and restores disc height and root canal dimensions, as well as load bearing ability of the anterior structures. Successful interbody construct reduces the postoperative segmental mobility and permits better graft incorporation ${ }^{[2,6]}$. The bilateral posterior lumbar interbody fusion (PLIF) procedure was first introduced by Cloward for lumbar interbody fusion and neural decompression. In addition, the interbody space has more vascularity than the posterolateral space, increasing the potential for a solid fusion mass to form, We report our result of using PLIF for the management of spondylolisthesis. Postoperatively, the differentiation between a fusion and a pseudo-arthrosis is easier after an interbody fusion. There is clear evidence that circumferential fusions have significantly higher radiographic fusion rates. The circumferential fusions can be performed via posterior approach as in a 
posterior lumbar interbody fusion (PLIF) and transforaminal lumbar interbody fusion (TLIF), or as combined posterior and anterior approach, as used in anterior lumbar interbody fusion (ALIF).

Harms and Jeszensky developed the TLIF technique, in which bone graft and titanium mesh were placed via a posterolateral transforaminal route into a distracted disc space in conjunction with a supplemental pedicle screw construct. The pedicle screws can be used for dynamic restoration of lumbar lordosis by using the interbody spacer as a cantilever. This was a variation of the posterior lumbar interbody fusion (PLIF) technique first described by Cloward.

The PLIF has several advantages over ALIF. Because PLIF does not require anterior abdominal exposure, it avoids all of the vascular, abdominal wall, and autonomic complications of ALIF. It is a single-stage procedure unlike ALIF requiring posterior fusion. Consequently, PLIF can be performed safely above L-3 with less risk of conus medullaris retraction and injury, and is better suited for revision cases with significant epidural fibrosis.

The purpose of this prospective study is to document our early experience using the PLIF procedure and to evaluate if PLIF is a safe and effective technique for lumbar interbody fusion in spondylolisthesis.

\section{AIMS and objectives of study}

In this Prospective Study my aim of the study is to asses:-

1. Radiological outcome in cases of posterior lumbar interbody fusion in lumbar Spondylolisthesis.

2. Clinicaloutcome like postoperative pain and neurological claudication assessment subjectively.

\section{Materials and methods \& observation}

A Prospective hospital based clinical study, conducted during the period of October 2016 - April 2018, for a period of 18 months, at hospital in Bengaluru, where 30 patients are selected based on inclusion and exclusion criteria.
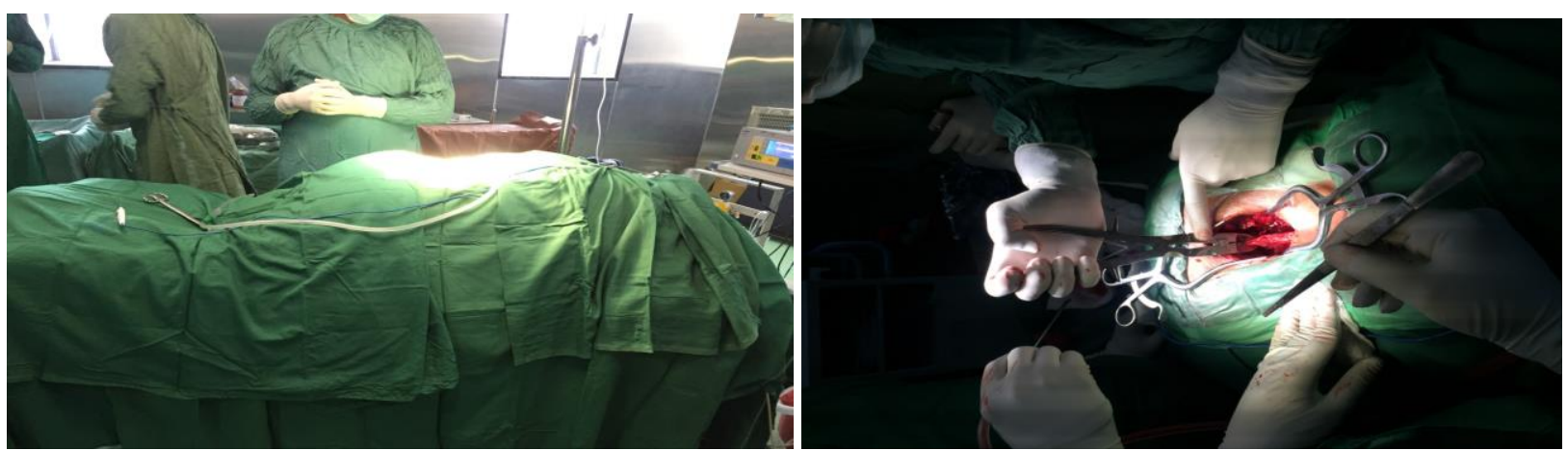

Stabilization with Rods
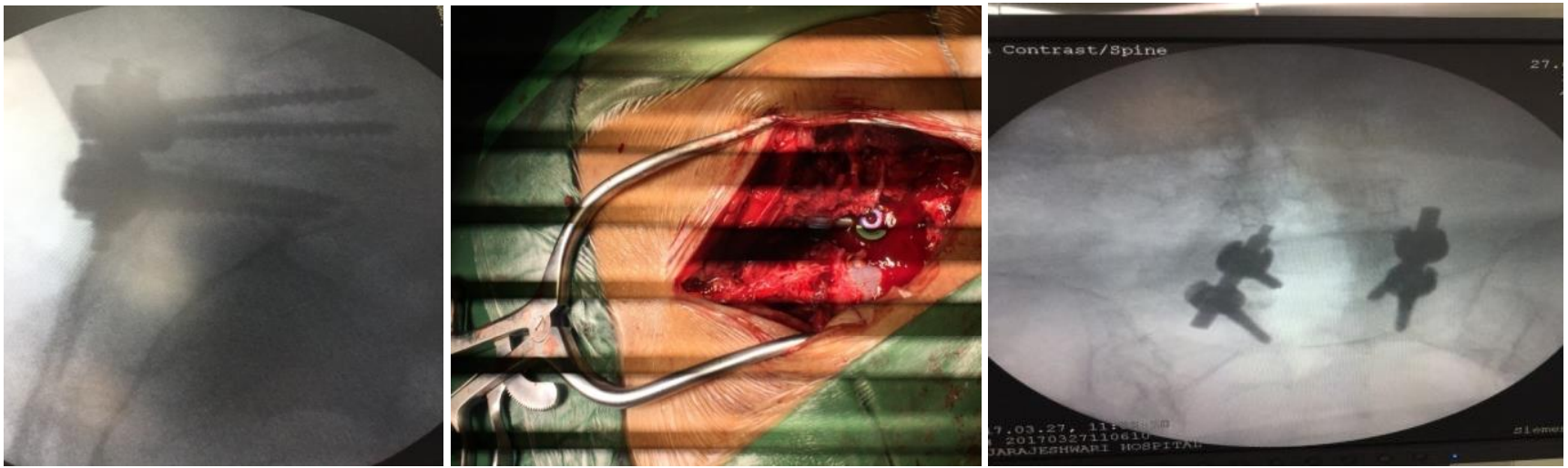

Fig 1: Age distribution of patients are studied

In present study all the patients are between the age of 20-70 years as shown in the table 1 majority of the patients are in age group between 51-60 yrs with a mean age group of $52.63 \pm 12.10$ there is one patient who is less than 30 years and six patients with more than 60 years.

Table 1: In present study all the patients are between the age of 2070 years

\begin{tabular}{|c|c|c|}
\hline Age in years & No. of patients & \% \\
\hline$<30$ & 1 & 3.3 \\
\hline $30-40$ & 5 & 16.7 \\
\hline $41-50$ & 6 & 20.0 \\
\hline $51-60$ & 12 & 40.0 \\
\hline $61-70$ & 6 & 20.0 \\
\hline Total & 30 & 100.0 \\
\hline
\end{tabular}

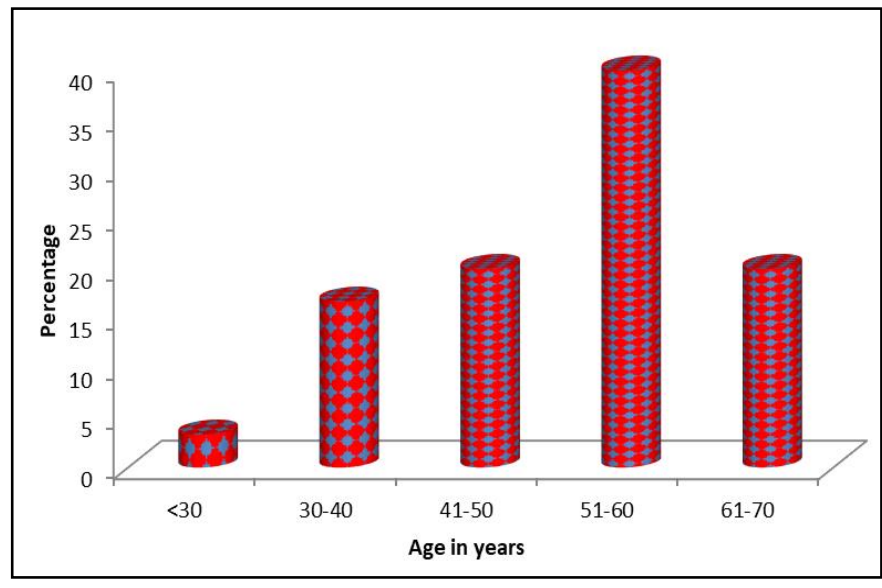

Graph 1: As depicted in the graph above 
Table 2: Gender distribution of patients is studied

\begin{tabular}{|c|c|c|}
\hline Gender & No. of patients & \% \\
\hline Female & 20 & 66.7 \\
\hline Male & 10 & 33.3 \\
\hline Total & 30 & 100.0 \\
\hline
\end{tabular}

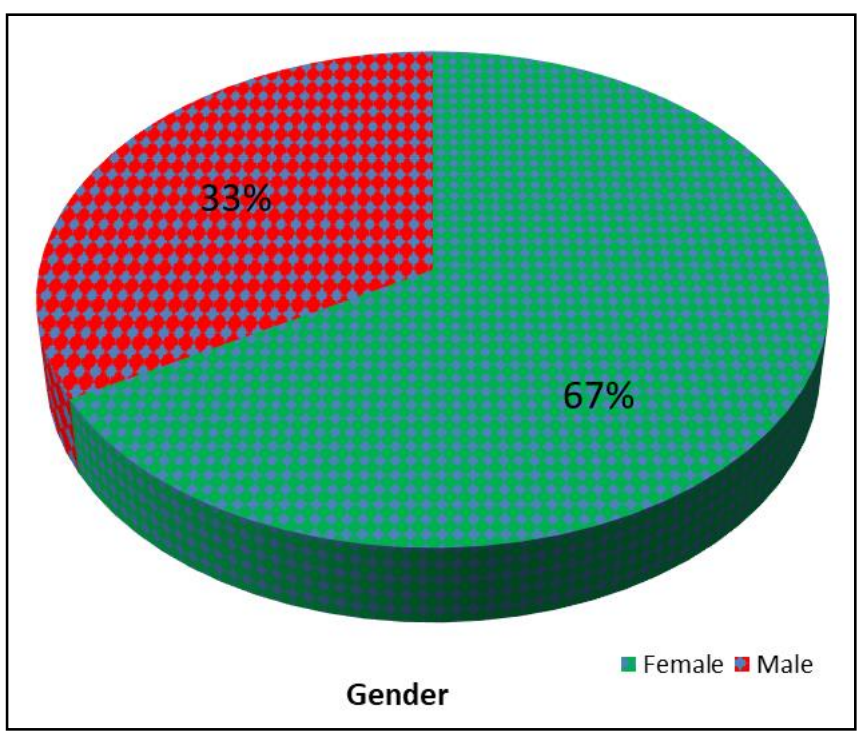

Graph 2: As depicted female and male

In the study it was observed that out of total patient studied that $20(66.7 \%)$ were female and $10(33.3 \%)$ were male, most commonly involved were female and indicates incidence is more common in females. As depicted in graph 2.

\section{Discussion}

Lumbar Spondylolisthesis is a common cause for lower-back pain, radiculopathy, and neurogenic claudication among the adult population, The ideal surgical treatment remains controversial.

Traditionally, the surgical management of degenerative lumbar spondylolisthesis consisted of decompressive laminectomy alone. In patients who had a radical decompressive laminectomy in which all of the facet joints were removed, the outcome was poor and listhesis progressed.

\section{Conclusion}

Decompression primarily relieves radicular symptoms and neurogenic claudication whereas fusion primarily relieves back pain by elimination of instability. The addition of posterofusion instrumentation (Pedicle screws and cage) enhances the ability to obtain a solidarthrodesis, Posterior Lumbar interbody fusion is a excellent procedure for lumbar spondylolisthesis achieved a significant reduction in low back pain and claudication pain and significant amount of radiological reduction and union.

\section{References}

1. Boos N, Marcheri D, Zuber K, Aebi M. Treatment of severe spondylolisthesis by reduction and pedicular fixation: A 4-6 year follow up study. Spine. 1993; 18:1655-1661.

2. Brantigan JW, Neidre A. Achievement of normal saggital plane alignment using a wedged carbon fibre reinforced polymer fusion cage in treatment of spondylolisthesis. Spine J, 2003.

3. de Loubress CG, Bon T, Deburge A, Lassale B, Benoit M Posterolateral fusion for radicular pain in isthmic spondylolisthesis. Clin Orthop. 1996; 323:194-201.
4. Madan S, Boeree NR. Outcome of posterior lumbar interbody fusion versus posterolateral fusion for spondylolytic spondylolisthesis. Spine. 2002; 27:15361542.

5. Rubeli MU, Grob D. Fusion of spondylolisthesis. SpineState Art Rev. 1992; 6:577-592.

6. Kwon KK, Scott B, Scott DD, Vaccaro A, Hilibrand A et al. Radiographic analysis of transforaminal lumbar interbody fusion for the treatment of adult isthmic spondylolisthesis. J Spinal Disord Tech. 2003; 16:469476. 